\title{
Détermination des puissances spécifiques critiques dans des rivières à charge de fond caillouteuse
}

Determination of critical unit stream power in gravel-bed rivers

Bestimmung der spezifischen kritischen Kräfte in Flüssen mit kieseliger

Grundfracht

\section{François Petit, Geoffrey Perpinien et Carl Deroanne}

\section{(2) OpenEdition}

Édition électronique

URL : http://journals.openedition.org/rge/4203

DOI : $10.4000 /$ rge.4203

ISSN : 2108-6478

Éditeur

Association des géographes de l'Est

Édition imprimée

Date de publication : 1 janvier 2000

ISSN : 0035-3213

Référence électronique

François Petit, Geoffrey Perpinien et Carl Deroanne, « Détermination des puissances spécifiques critiques dans des rivières à charge de fond caillouteuse », Revue Géographique de l'Est [En ligne], vol. 40 / 1-2 | 2000, mis en ligne le 06 août 2013, consulté le 08 septembre 2020. URL : http:// journals.openedition.org/rge/4203; DOI : https://doi.org/10.4000/rge.4203

Ce document a été généré automatiquement le 8 septembre 2020.

Tous droits réservés 


\title{
Détermination des puissances spécifiques critiques dans des rivières à charge de fond caillouteuse
}

\author{
Determination of critical unit stream power in gravel-bed rivers \\ Bestimmung der spezifischen kritischen Kräfte in Flüssen mit kieseliger \\ Grundfracht
}

François Petit, Geoffrey Perpinien et Carl Deroanne

\section{Introduction}

Parmi les critères de mise en mouvement de la charge de fond, la vitesse critique d'érosion, introduite par Hjulstrôm (1935) est la notion la plus ancienne et probablement la plus utilisée encore à l'heure actuelle. Toutefois, l'utilisation de la force tractrice se généralise de plus en plus en géomorphologie, spécialement depuis que des valeurs de forces tractrices critiques ont été proposées pour la charge caillouteuse, prenant en compte, grâce au critère adimensionnel de Shields ${ }^{1}$, l'effet de saillie relative et d'imbrication (Andrews, 1983). L'utilisation des forces tractrices permet en outre de faire des estimations du transport solide, par le biais de l'équation de Meyer-Peter ${ }^{2}$. Toutefois, dans les estimations de transport solide, il convient généralement de mettre en œuvre des approches différentes de façon à pouvoir confronter les résultats. C'est dans la perspective d'une évaluation du transport solide que Bagnold (1977) a initialement introduit la notion de puissance spécifique; depuis lors, elle a trouvé d'autres champs d'application, principalement en vue d'appréhender l'activité des rivières, notamment en ce qui concerne les formes et la dynamique des méandres (Ferguson, 1981 ; Bravard, 1991) ou la possibilité de réaction et d'adaptation des rivières en réponse à des travaux d'aménagement (Brookes, 1988). Par ailleurs, la puissance spécifique est maintenant utilisée couramment comme facteur de 
discrimination des lits fluviaux, notamment pour l'apparition du tressage (Van den Berght, 1995). Plus récemment, la distance que parcourt la charge de fond une fois mobilisée a été mise en relation avec l'excès de puissance spécifique par rapport à la puissance spécifique critique de mise en mouvement (Hassan et al., 1993 ; Gintz et al., 1996). Toutefois, le problème majeur réside dans le fait qu'il n'existe pas de relation unique qui lie la puissance spécifique critique à la taille du matériel mobilisé. Certes Bagnold a proposé une relation, assez complexe d'application pratique, mais différents auteurs ont remis cette relation en question.

\section{Rappel des équations}

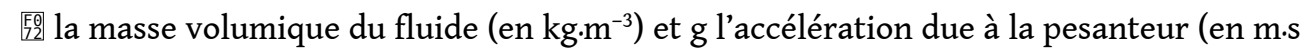
${ }^{-2}$ ). Afin de permettre des comparaisons entre rivières différentes, on retient généralement le débit à pleins bords, auquel cas w est la largeur prise entre les berges au niveau du débordement. Notons que dans les rivières à chenaux tressés, où il est difficile de définir un débit à pleins bords, on retient généralement la valeur du débit de récurrence 1,5 ans, ce qui est assez proche de la récurrence du débit à pleins bords, même si des nuances doivent être apportées en fonction notamment de la nature de la charge de fond et du mode d'alimentation des rivières (Petit \& Pauquet, 1997). faute de mieux, comme la pente du plan d'eau. Mais, la puissance doit être considérée comme un descripteur assez sommaire, facile d'emploi en l'absence de données précises, et, de ce fait, sur de longs tronçons de rivières on peut considérer $\mathrm{S}$ comme étant la pente longitudinale du lit, voire même de la plaine alluviale. suivante :

avec 圈 est la force tractrice totale exprimée en $\mathrm{N} \cdot \mathrm{m}^{-2}$ et $\mathrm{u}$ la vitesse moyenne du courant exprimée en $\mathrm{m} \cdot \mathrm{s}^{-1}$. Rappelons que la force tractrice totale est en réalité la somme de deux composantes: (i) la force tractrice due à la résistance des grains (la grain shear stress 医'), la seule qui doit être prise en compte dans la mobilisation et le transport de la charge de fond; (ii) une force tractrice supplémentaire due à la résistance des formes $\mathrm{du}$ lit (la bedform shear stress (5耳"). Comme on le verra par la suite, il conviendrait de tenir compte de cette distinction, même lorsque les puissances spécifiques seront utilisées.

Bagnold (1980) a proposé une équation qui permet de déterminer la puissance spécifique de mise en mouvement (59) à partir de laquelle les sédiments commencent à

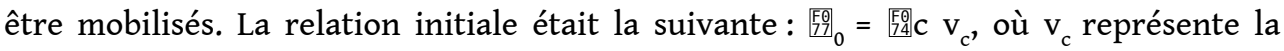
vitesse critique de mise en mouvement et tc la force tractrice critique pour laquelle il faisait intervenir le critère adimensionnel de Shields $\left({ }_{\left[i_{1}\right.}\right)$ avec une valeur égale à 0,040 :

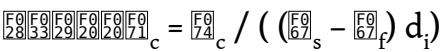




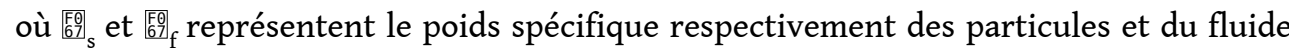
et $d_{i}$ la taille des particules (en $\mathrm{m}$ ). Retenant une densité des particules égales à 2,689 $\mathrm{g}$.

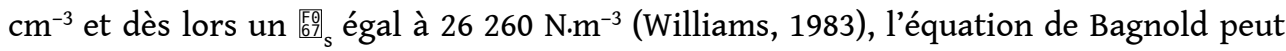
s'exprimer de la façon suivante :

$$
\text { [Fi }
$$

où 诓 taille des particules (en $\mathrm{mm}$ ).

Des relations qui lient la puissance spécifique critique à la seule taille du matériau (sans prendre en compte la profondeur), ont été proposées par la suite. A partir d'observations effectuées dans des rivières à forte pente du Colorado, Costa (1983) a mis en évidence une relation, applicable essentiellement à des éléments grossiers (supérieurs à $50 \mathrm{~mm}$ et allant jusqu'à $1000 \mathrm{~mm}$ ) :

[Fo:

(où 夢 ${ }_{0}$ est exprimé en W. $\mathrm{m}^{-2}$ et $\mathrm{d}_{\mathrm{i}}$ en $\mathrm{mm}$ ). Cette relation est obtenue par régression, la droite passant au milieu du nuage de points ; toutefois Costa a également proposé, sur base des mêmes observations, une courbe enveloppe qui passe sous les points, tout en conservant une pente parallèle à la droite obtenue par régression :

[F⿱

Toujours pour des éléments grossiers (entre 10 et $1500 \mathrm{~mm}$ ), Williams (1983) reprenant une série d'observations publiées dans la littérature, a proposé la relation :

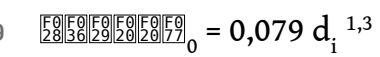

Finalement, comme cela apparaît à la figure 1, ces deux dernières relations donnent des résultats assez semblables. Toutefois, elles sont loin d'être généralisables, puisque Reid \& Frostick (1985) observent dans une rivière de dimension modeste du sud de l'Angleterre $\left(\mathrm{Q}_{\mathrm{b}}=19,6 \mathrm{~m}^{3} \cdot \mathrm{s}^{-1}\right)$ que la puissance spécifique doit atteindre 35 à $40 \mathrm{~W} \cdot \mathrm{m}^{-2}$ pour déstabiliser un pavage constitué d'éléments de $22 \mathrm{~mm}$. D'autre part, Assani (1997) trouve, dans une rivière ardennaise, des valeurs du même ordre de grandeur que ces derniers auteurs ; la puissance spécifique doit en effet atteindre $26 \mathrm{~W} \cdot \mathrm{m}^{-2}$ pour mettre en mouvement des éléments dont le $\mathrm{D}_{50}=28 \mathrm{~mm}$. Enfin, Blizard \& Wohl (1998) ont mis en relation la puissance spécifique et le $\mathrm{D}_{84}$ connu grâce à des prélèvements effectués avec un échantillonneur de charge de fond. Comme le soulignent ces auteurs, les relations ne sont pas très significatives mais nous pouvons néanmoins épingler les

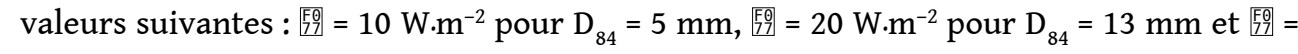
$25 \mathrm{~W} \cdot \mathrm{m}^{-2}$ pour $\mathrm{D}_{84}=16 \mathrm{~mm}$. Il convient de souligner que ces mesures ont été effectuées dans une rivière située en tête de bassin (superficie inférieure à $10 \mathrm{~km}^{2}$ ), caractérisée par un fort gradient, par une organisation en step-pool system ${ }^{3}$ et par la formation de nombreux embâcles, c'est à dire dans des systèmes à forte dissipation d'énergie due à la résistance des formes et à une rugosité importante. 
Fig. 1 : Relations entre la puissance spécifique critique (w0 en W. m-2) et la taille des éléments (en $\mathrm{mm})$

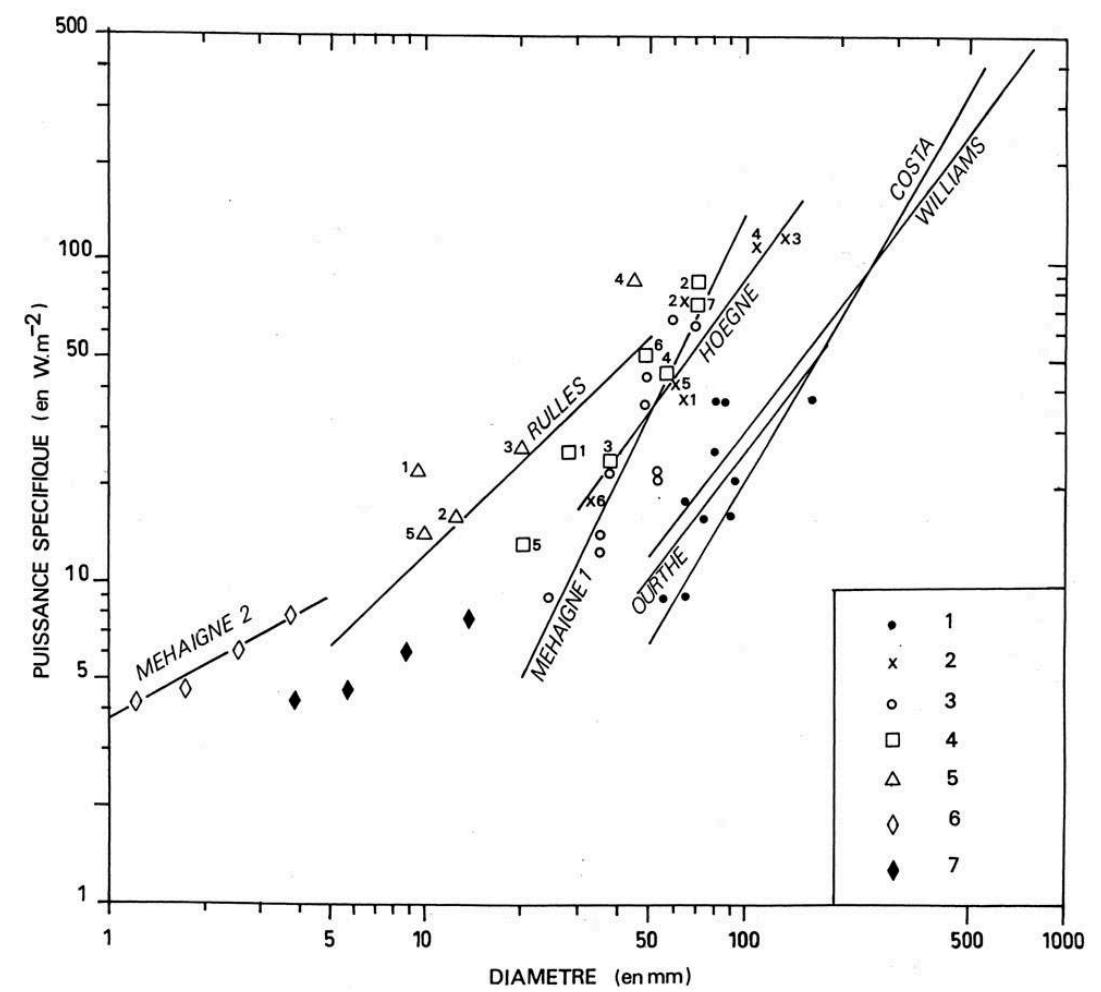

Observations effectuées dans : (1) l'Ourthe, (2) la Hoëgne (les chiffres renvoient aux stations de mesures, prises d'amont en aval), (3) la Mehaigne (avec placettes de marquage), (4) dans des rivières de taille intermédiaire, les chiffres renvoient aux différentes rivières avec 1 la Warche, 2 la Magne, 3 la Berwinne, 4 la Lhomme, 5-7 la Lesse ; (5) dans des têtes de bassin, les chiffres renvoient aux différentes rivières avec 1 et 2 le Ru des Waidages (avant et après enlèvement des embâcles), 3 le Ruisseau de la Mer, 4 le Ruisseau de Belleva, 5 le Ruisseau de Wavelinse ; (6) dans la Mehaigne avec un échantillonneur de charge de fond (avec le $D_{50}$ du matériel prélevé) ; (7) dans la Mehaigne avec un échantillonneur de charge de fond (avec le $\mathrm{D}_{84}$ du matériel prélevé).

Les valeurs présentées ci-dessus s'écartent nettement de celles déduites des relations de Costa et Williams. Il semblait donc utile d'affiner les valeurs des puissances spécifiques de mise en mouvement, pour des rivières de dimension modeste, à pente moyenne, caractérisées par une charge caillouteuse de taille intermédiaire.

\section{III. méthodologie et caractéristiques des rivières Etudiées}

Les rivières étudiées, situées pour la plupart en Ardenne, ont toutes une charge caillouteuse et une pente assez marquée, variant de plus de 50 pour mille pour les rivières en tête de bassin à moins de 2 pour mille pour les rivières les plus importantes (tableau 1). Le régime des débits est relativement contrasté car ces rivières sont développées sur un substratum imperméable (quartzites et quartzophyllades du Dévonien inférieur). Toutefois, quelques-unes de ces rivières sont partiellement développées sur des calcaires. 
Tableau 1 : Caractéristiques des rivières étudiées.

\begin{tabular}{|c|c|c|c|}
\hline & $\begin{array}{l}\text { Superficie } \\
\text { du BV }\left(\mathrm{km}^{2}\right)\end{array}$ & $\begin{array}{l}\text { Pente }{ }^{(*)} \\
\left(m \cdot m^{-1}\right)\end{array}$ & Réf. \\
\hline Ru des Waidages & 0,3 & 0,046 & Assani (1991) \\
\hline Ruisseau de la Mer & 1,4 & 0,071 & Mercenier (1973) \\
\hline Ruisseau de Belleva & 12,5 & 0,049 & Mercenier (1973) \\
\hline Ruisseau de Wavelinse & 4,3 & 0,024 & Dave (1974) \\
\hline Rulles & 16,2 & 0,012 & Petit (1987) \\
\hline Magne & 41,8 & 0,016 & Pironet (1995) \\
\hline Hoëgne & 219 & 0,017 & Deroanne (1995) \\
\hline Mehaigne & 354 & 0,0021 & Perpinien (1998) \\
\hline Berwinne & 118 & 0,0039 & Parotte (1991) \\
\hline Warche & 118 & 0,0042 & Assani (1997) \\
\hline Lhomme & 474 & 0,0022 & Franchimont (1993) \\
\hline Lesse (1) & 419 & 0,0028 & Franchimont (1993) \\
\hline Lesse (2) & 1090 & 0,0020 & Franchimont (1993) \\
\hline Ourthe inf. & 2660 & 0,0025 & Petit et al. (1996) \\
\hline
\end{tabular}

(*) Pente calculée de la source à la station considérée.

Des campagnes de marquage de la charge de fond ont été effectuées dans des rivières de taille différente et ont été complétées par des traitements réalisés à partir d'observations effectuées précédemment dans différentes rivières de haute Belgique. Les plus petites de ces rivières ont un bassin versant de quelques kilomètres carrés, la plus grande atteint près de $2700 \mathrm{~km}^{2}$. Ces observations ont été mises en relation avec des paramètres dynamiques, selon une méthodologie comparable mais qui a cependant nécessité certaines adaptations, notamment en fonction de la dimension des rivières. Dans la plupart des rivières, les marquages colorimétriques ont été effectués in situ afin de ne pas perturber l'agencement du matériel constituant le lit. Les caractéristiques du matériel ont été estimées par la méthode de la grille, méthode proposée par Kellerhals \& Bray (1971) et appliquée avec succès notamment dans le Canal de Miribel (Poinsart, 1992). Des repérages topographiques ont été réalisés afin de s'assurer qu'il y avait bien eu mise en mouvement et non enfouissement du matériel. Dans les rivières de dimension plus modeste, la technique de la grille s'est avérée difficile d'application étant donné que les bancs de galets n'affleuraient que sur des portions trop restreintes du lit. La taille du matériel mobilisé a alors été estimée soit en mesurant des éléments individuels préalablement marqués in situ, soit à partir de prélèvements effectués dans des pièges à sédiments.

Des campagnes de mesures plus intensives ont été réalisées sur quatre rivières: l'Ourthe, la Mehaigne, la Hoëgne et la Rulles, ce qui a permis de tracer des droites de régressions pour chacune d'elles.

\section{Résultats et discussion}

L'essentiel des résultats sont repris à la figure 1. Pour les quatre rivières où des relations ont été mises en évidence, les équations sont présentées au tableau 2. Quelques précisions doivent être apportées à propos de la manière dont ces résultats ont été obtenus. 


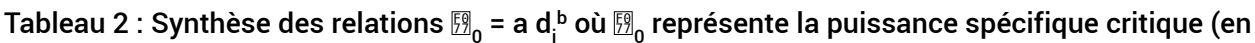
$W \cdot \mathrm{m}^{-2}$ ) et $\mathrm{d}_{\mathrm{i}}$ la taille du matériel mobilisé (en $\mathrm{mm}$ )

\begin{tabular}{|l|c|c|c|c|c|c|}
\hline & $\mathrm{a}$ & $\mathrm{b}$ & $\mathrm{n}$ & $\mathrm{r}$ & G.M (*) & $\begin{array}{r}\text { Réf. } \\
(\mathrm{mm})\end{array}$ \\
\hline Ourthe inf. & 0,054 & 1,340 & 10 & 0,70 & $55-160$ & Petit et al., 1996 \\
Hoëgne & 0,192 & 1,331 & 6 & 0,94 & $32-160$ & Deroanne, 1995 \\
Mehaigne (1) & 0,011 & 2,051 & 10 & 0,89 & $25-70$ & Perpinien, 1998 \\
Mehaigne (2) & 3,738 & 0,559 & 4 & 0,98 & $1-5$ & Perpinien, 1998 \\
Rulles & 1,374 & 0,963 & 26 & 0,90 & $5-60$ & Petit (en prép.) \\
\hline
\end{tabular}

(*) G.M = gamme de matériau analysé ; a et b représentent respectivement le coefficient et l'exposant dans la relation 䍐 ${ }_{0}=a \mathrm{~d}_{\mathrm{i}}^{\mathrm{b}}, \mathrm{r}$ le coefficient de corrélation et $\mathrm{n}$ le nombre de cas intervenant dans la régression.

- Dans le cas de l'Ourthe, sept placettes ont été marquées dans trois sites différents, se présentant comme de vastes seuils obliques. La mise en mouvement de tous les éléments constituant les placettes a été observée pour quatre crues différentes. La relation entre le $\mathrm{D}_{50}$ mobilisé et la puissance spécifique est médiocre, ce qui tient probablement au fait que les sites sont relativement hétérogènes et qu'il s'agit ici de valeur de puissances intégrée à l'ensemble de la section transversale alors qu'il existe, au sein même des unités morphologiques, des différences assez sensibles. Ce point avait déjà été évoqué dans l'analyse des forces tractrices critiques effectuées dans les même sites (Petit et al., 1996). Par ailleurs, comme il ressort de la figure 1, les valeurs de puissance spécifique critique mises en évidence dans l'Ourthe se rapprochent de celles déduites des relations de Costa et de Williams et s'écartent sensiblement de l'équation de Bagnold puisque par exemple, en appliquant cette dernière relation, $\mathrm{w}$ devrait dépasser $90 \mathrm{~W} \cdot \mathrm{m}^{-2}$ pour mobiliser un $\mathrm{D}_{50}=50 \mathrm{~mm}$ sous une profondeur de $2 \mathrm{~m}$.

- Sept sites de la Hoëgne, échelonnés selon un linéaire établi à partir de la source jusqu'à sa confluence, ont été marqués en utilisant la technique de la grille. Cette rivière possède un gradient marqué, puisque la dénivellation est de $520 \mathrm{~m}$ sur une distance de $31 \mathrm{~km}$. On observe une décroissance assez brusque de la taille du matériel : il dépasse $200 \mathrm{~mm}$ en amont et n'atteint plus que $50 \mathrm{~mm}$ dans la partie aval du cours (Deroanne, 1995). Une mobilisation du matériel a été observée pour deux crues (représentant respectivement $0,3 Q_{b}$ et $0,6 Q_{b}$ à la station de référence). La puissance $a$ été calculée à chacun des sites de marquage, en fonction de débits spécifiques préalablement testés ainsi que des pente et largeur locales. La première de ces crues, la plus faible, n'a affecté que les sites d'aval (points 5 et 6) où la superficie du bassin versant est supérieure à $80 \mathrm{~km}^{2}$, mais pas les sites d'amont. En revanche, la seconde crue a également affecté les sites d'amont où la superficie du bassin versant est inférieure à une dizaine de $\mathrm{km}^{2}$.

28 - Des marquages ont été effectués dans cinq sites différents de la Mehaigne, s'échelonnant également sur un linéaire amont-aval mais qui dans ce cas représente seulement une quinzaine de kilomètres (Perpinien, 1998). La mobilisation des placettes a été observée dans chacun des sites, ceci à deux reprises (crues valant respectivement $0,3 Q_{b}$ et $0,8 Q_{b}$ à la station de référence); les $D_{50}$ mobilisés varient de $25 \mathrm{~mm}$ à $70 \mathrm{~mm}$. La relation établie est assez proche de celle mise en évidence sur la Hoëgne. Toujours sur la Mehaigne, des prélèvements ont été effectués à l'aide d'un échantillonneur de charge de fond, lors de crues plus faibles que les crues qui ont mobilisé la charge caillouteuse. Ceci tient à la disposition géographique du bassin : sa partie supérieure se 
trouve exclusivement sur des sables tertiaires, le Paléozoïque, qui conditionne l'apparition de la charge caillouteuse, n'affleurant que dans la partie aval du cours; il existe ainsi un charriage d'éléments fins lors de crues de faible importance. Le nombre de points est certes limités (4) mais le coefficient de détermination est particulièrement bon $\mathrm{r}^{2}=0,963$, le $\mathrm{D}_{50}$ variant de $1 \mathrm{~mm}$ à $5 \mathrm{~mm}$. Comme il ressort de la figure 1 , cette relation s'écarte quelque peu des autres relations, mais rappelons que le mode d'identification de la taille des particules mobilisées est différent. Si, à l'instar de Blizard \& Wohl (1998), nous prenons le $\mathrm{D}_{84}$, ce qui peut se justifier puisqu'un échantillonneur de charge de fond a été utilisé, on note alors que les valeurs se disposent dans le prolongement des autres relations.

- La relation mise en évidence sur la Rulles est basée sur l'analyse détaillée de la mise en mouvement et du transport d'un nombre important d'éléments individuels : près de 270 cailloux marqués in situ lors de six campagnes de marquages différentes couvrant des crues allant du débit à pleins bords à une crue quinquennale. Ces observations ont été regroupées en 26 classes, chaque classe représentant le diamètre moyen de plusieurs éléments (une dizaine en moyenne) mobilisés ou transportés dans un site précis, pour une crue donnée. Précisons d'emblée que nous n'avons pas vu de différences sensibles entre les valeurs de mise en mouvement et celles assurant le transport, montrant qu'il n'y a pas d'effet de pavage et que les phénomènes d'imbrication sont peu développés. La puissance a été calculée aux différents profils là où il $\mathrm{y}$ a eu mobilisation ou transport du matériel; cette évaluation a pu se faire à chaque profil car la force tractrice et la vitesse moyenne du courant ont été mesurées à chacun d'eux, pour les gammes de débits mobilisateurs (Petit, 1987).

Précisons tout d'abord que la relation établie dans la Rulles est basée sur des points se situant principalement entre 5 et $40 \mathrm{~mm}$ (seuls quelques points présentent des diamètres supérieurs), alors que dans les autres relations la taille des éléments est nettement plus importante. Mais indépendamment de ce fait, cette relation apparaît très différente de celles mises en évidence dans les autres rivières. En effet, à diamètre égal, la puissance spécifique de mise en mouvement est systématiquement plus élevée. Ceci s'explique par le fait que cette relation a été établie dans une rivière de dimension modeste, caractérisée par un méandrage marqué, par une alternance rapprochée entre les seuils et les mouilles et par de vastes zones de contre-courant. Or, on sait qu'une grande partie de l'énergie de la rivière est consommée pour vaincre les résistances de

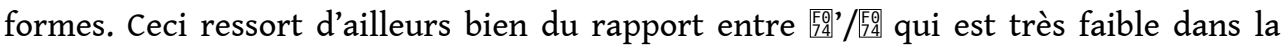
Rulles ( 0,15 à 0,3 , pour le débit à pleins bords), impliquant qu'une grande part de la

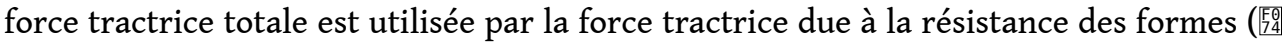
"), ce qui limite la force tractrice due à la résistance des grains (蜸') et donc l'énergie disponible pour la mise en mouvement et le transport de la charge de fond (Petit, 1990).

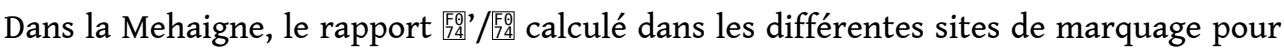
les crues mobilisatrices, est plus élevé $(0,45)$. Il en est de même dans la Hoëgne où il varie de 0,10 à 0,50 en fonction de la position par rapport à la distance à la source (Deroanne, 1995). Dans l'Ourthe ce rapport est de 0,65 à 0,7 pour le débit à pleins bords. Ceci prouve que lorsque la rugosité totale et donc les pertes d'énergie dues aux formes diminuent, la puissance spécifique de mise en mouvement est, à diamètre égal, elle aussi plus faible.

31 Les valeurs de puissance critiques mises en évidence dans des études plus ponctuelles confirment cette interprétation. En effet, dans ces résultats, il faut distinguer deux 
catégories de rivières : les premières sont des rivières situées en tête de bassins, les secondes ont une taille nettement plus importante (tableau 1).

Comme il ressort de la figure 1 , les puissances spécifiques critiques de mise en mouvement observées dans les têtes de bassin sont élevées et se rapprochent de celles mises en évidence dans la Rulles. Quelques précisions doivent être apportées à propos de ces études. Des expériences ont été réalisées par Assani (1991) dans le Ru des Waidages; ce thalweg d'ordre 2 se présente en réalité comme un drain où prolifèrent de nombreux embâcles. La puissance de mise en mouvement a été estimée à partir du déplacement de plus d'une soixantaine de cailloux marqués. Ensuite, les embâcles ont été détruits, on a laissé le fond se reconstituer et une seconde campagne de marquage a été effectuée afin d'estimer la puissance de mise en mouvement dans un système où la rugosité a nettement diminué. Le rapport 頤/䍇 dépasse à peine 0,10 dans le système avec embâcles et atteint 0,35 lorsque les embâcles ont disparu (Assani \& Petit, 1995). Ceci montre que les pertes d'énergie due à la résistance des formes restent appréciables et justifient des puissances critiques plus élevées. Les rivières étudiées par Mercenier (1973) et par Dave (1975) sont elles aussi caractérisées par de nombreux embâcles végétaux voire même par des affleurements de blocs, éléments qui contribuent à accroître la rugosité et donc des pertes d'énergie dues à la résistance des formes. Les puissances déduites des mesures effectuées par ces auteurs se rapprochent de la relation mise en évidence dans la Rulles.

Les puissances critiques de mise en mouvement observées dans des rivières de taille intermédiaire se rapprochent des relations mises en évidence dans la Hoëgne et la Mehaigne. Dans ces rivières, les pertes d'énergie liées à la résistance des formes restent importantes par rapport à l'Ourthe, mais moindres que dans les ruisseaux situés en tête de bassin. Ainsi, dans la Berwinne, le rapport 卧/䍇 varie de 0,3 à 0,5 pour le débit à pleins bords (Parotte, 1991); dans la Lhomme et la Lesse, rivières plus importantes avec des bassins versants atteignant $500 \mathrm{~km}^{2}$ avant leur confluence, ce rapport est de l'ordre de 0,45 à 0,6 (Franchimont, 1994).

Étant donné l'assez faible dispersion des points qui apparaît bien à la figure 1, les valeurs mises en évidence dans des rivières de taille intermédiaire ont été intégrées aux résultats obtenus dans la Hoëgne et la Mehaigne, afin d'en déduire une relation unique. Celle-ci se présente sous la forme suivante :

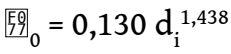

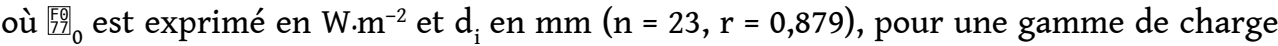
de fond dont le diamètre est compris entre 20 et $150 \mathrm{~mm}$, dans des rivières dont la superficie du bassin varie entre 40 et $500 \mathrm{~km}^{2}$. 
Tableau 3 : Relation entre le matériel mobilisé et la puissance spécifique.

\begin{tabular}{|c|c|c|c|c|}
\hline & $\underset{(\mathrm{mm})}{\mathrm{d}_{\mathrm{i}}}$ & $\begin{array}{c}\omega_{0} \\
\left(W / m^{2}\right)\end{array}$ & Matériel & Réf. \\
\hline \\
\hline 1. Ru des Waidages (a) & 9,7 & 21,5 & (cailloux marqués) & Assani (1991) \\
\hline 2. (b) & 12,5 & 15,3 & (cailloux marqués) & Assani (1991) \\
\hline 3 R. de la Mer & 20 & 27 & (piège à sédiments) & Mercenier (1973) \\
\hline 4. R. de Belleva & 45 & 80,4 & (cailloux marqués) & Mercenier (1973) \\
\hline 5. R. de Wavelinse & 9,8 & 14 & (piège à sédiments) & Dave (1975) \\
\hline Rivières de taille intermédiaire & & & & \\
\hline 1. Warche & 28 & 26 & (placettes cx marqués) & Assani (1997) \\
\hline 2. Magne & 70 & 84 & (placettes cx marqués) & Pironet (1995) \\
\hline 3. Berwinne & 38 & 23 & (cailloux marqués) & Parotte (1991) \\
\hline 4. Lhomme & 58 & 45 & (placettes cx marqués) & Franchimont (1994) \\
\hline 5. Lesse Eprave & 20 & 14 & (placettes cx marqués) & Franchimont (1994) \\
\hline 6. Lesse & 48 & 52 & (placettes cx marqués) & Franchimont (1994) \\
\hline 7. Lesse & 66 & 76 & (placettes cx marqués) & Franchimont (1994) \\
\hline
\end{tabular}

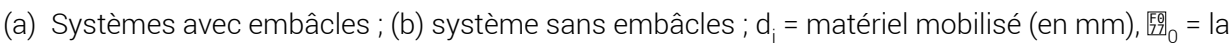
puissance spécifique (en $\left.\mathrm{W} \cdot \mathrm{m}^{-2}\right)$.

\section{Conclusions}

Trois types de relations liant la puissance spécifique critique $\left(\right.$ 湢 $\left._{0}\right)$ à la taille des éléments mobilisés $\left(\mathrm{d}_{\mathrm{i}}\right)$ ont été mises en évidence. Pour la rivière la plus importante (l'Ourthe), les valeurs de 眀 ${ }_{0}$ sont les plus faibles et se rapprochent des valeurs proposées par Costa et par Williams. Dans les rivières de dimension intermédiaire (taille du bassin comprise entre 40 et $500 \mathrm{~km}^{2}$ ), les puissances spécifiques critiques sont plus élevées, ce qui résulte du fait qu'il existe une résistance des formes plus importante, cette dernière consommant une part accrue de l'énergie, au détriment de celle disponible pour la mobilisation et le transport de la charge de fond. L'importance de cette résistance des formes est généralement appréhendée par la bedform shear

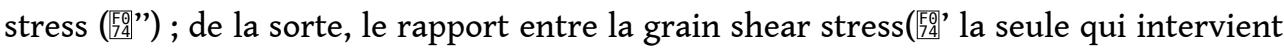
dans la mobilisation et le transport de la charge de fond), et la force tractrice totale (楺), peut être utilisé, a contario, comme un descripteur de cette perte d'énergie liée à la résistance des formes. Ce rapport varie entre 0,4 à 0,5 dans des rivières de dimension intermédiaire tandis qu'il atteint 0,7 dans l'Ourthe. Dans les têtes de bassin (superficie inférieure à $20 \mathrm{~km}^{2}$ ), où les pertes d'énergie dues à la résistance des formes sont

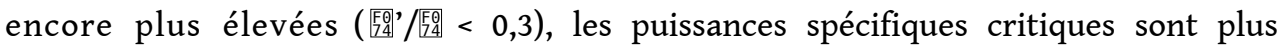
importantes que dans les deux autres types. Finalement, comme cela tend à se généraliser pour le critère de Shields, il conviendrait de faire la distinction entre une puissance spécifique critique brute (匰 0 prenant en compte la force tractrice totale) et une puissance spécifique critique propre aux grains (姫'), ce qui risque toutefois de restreindre le champ pratique d'application de la puissance spécifique, étant donné les informations supplémentaires que cette utilisation implique. 


\section{BIBLIOGRAPHIE}

Andrews E.D. (1983). - Entrainment of gravel from naturally sorted riverbed material. Geol. Soc. Am. Bull., 94, 1225-1231.

Assani A.A. (1991). - Influence des embâcles sur la morphologie et la dynamique d'un drain à charge caillouteuse (Hautes-Fagnes, Belgique). Mém. Maîtrise Géol. Terrains Superficiels, Univ. Liège, 129 p.

Assani A.A. (1997). - Recherche d'impacts d'une retenue sur le comportement d'une rivière ardennaise (Hydrologie, sédimentologie, morphologie et végétation); cas du barrage de Butchenbach sur la Warche (Belgique). Thèse Doctorat Sc. Géog., Univ. Liège, 482 p.

Assani A.A. \& Petit F. (1995). - Log-jams effect on bed-load mobility from experiments conducted in a small gravel-bed forest ditch, Catena, 25, 117 - 126.

Bagnold R.A. (1977). - Bed load transport by natural rivers. Water Resources Research, 13 (2), 303-312.

Bagnold R.A. (1980). - An empirical correlation of bedload transport rates in flumes and natural rivers. Proc. R.S. of London, A 372, 453-473.

Blizard C.R. \& Wohl E.E. (1998). - Relationships between hydraulic variables and bedload transport in a subalpine channel, Colorado Rocky Mountains, USA. Geomorphology, 22, 359-371. Bravard J.P. (1991). - La dynamique fluviale à l'épreuve des changements environnementaux : quels enseignements applicables à l'aménagement des rivières ? La Houille blanche, 7, 515-521.

Bravard J.P. \& Petit F. (1997). - Les cours d'eau, dynamique du système fluvial. A. Colin, Paris, 222 p.

Brookes A. (1988). - Channelized rivers : Perspecives for environmental managment. Wiley, 326p.

Costa J.E. (1983). - Palaeohydraulic reconstruction of flash-flood peaks from boulder deposits in the Colorado Front range. Geological Society of America Bulletin, 94 : 986-1004.

Dave C. (1975). - Étude de la dynamique fluviale d'un petit ruisseau de type torrentiel. Mém. Lic. Sc. géogr, Univ. Liège, $148 \mathrm{p}$.

Deroanne C. (1995). - Dynamique fluviale de la Hoëgne. Évaluation longitudinale des caractéristiques sédimentologiques du lit et des paramètres de mobilisation de la charge de fond. Mém. Lic. Sc. Géog., Univ. Liège, 155 p.

Ferguson R.I. (1981). - Channel forms and channel changes. In Lewin (Ed.) British rivers, London, G. Allen \& Unwin, 90-125.

Franchimont C. (1993). - Dynamique fluviale de la Lesse : fréquence des inondations, morphométrie des méandres et sédimentologie du lit. Mém. Lic. Sc. Géog., Univ. Liège, 121 p.

Gintz D., Hassan M.A. \& Schmidt K.H. (1996). - Frequency and magnitude of bedload transport in a mountain river. Earth Surface Processes and Landforms, 21, 433-455.

Hassan M.A., Church M. \& Ashworth P.J. (1992). - Virtual rate and mean distance of travel of individual clasts in gravel-bed channels. Earth Surface Processes and Landforms, 17, 617-627.

Hjulström P. (1935). - Studies of the morphological activity of rivers as illustraded by the River Fyris. Bull. Geol. Institute, University of Uppsala, 25, 221-527. 
Kellerhals R. \& Bray D.I. (1971). - Sampling procedures for coarse fluvial sediment, J. Hydraul. Div. ASCE, 97 (Hy 8), 1165-1180.

Mercenier J. (1973). - Dynamique fluviale dans un petit bassin du rebord méridional du plateau des Tailles. Mém. Lic. Sc. Géog., Univ. Liège, 148 p.

Parotte V. (1991). - Évolution géomorphologique actuelle d'une rivière en relation avec les modifications de son régime hydrologique. La Berwinne à Dalhem (Entre Vestre et Meuse). Mém. Lic. Sc. Géog., Univ. Liège, $84 \mathrm{p}$.

Perpinien G. (1998). - Dynamique fluviale de la Mehaigne : morphométrie, transports en solution et suspension, mobilisation de la charge de fond. Mém. Lic. Sc. Géog., Univ. Liège, 128 p.

Petit F. (1987). - The relationship between shear stress and the shaping of the bed of a pebbleloaded river (La Rulles-Ardenne). Catena, 14 (5), 453-468.

Petit F. (1990). - Evaluation of grain shear stress required to initiate movement of particle in natural rivers. Earth Suface Processes and Landforms, 15, 135-148.

Petit F., Pauquet A. \& Pissart A. (1996). - Fréquence et importance du charriage dans des rivières à charge de fond graveleuse. Géomorphologie, 2 (2) : 3-12.

Petit F. \& Pauquet A. (1997). - Bankfull discharge recurrence interval in gravel-bed rivers. Earth Surface Processes and Landforms, 22, 685-693.

Pironet C. (1995). - Régime hydrologique, transports de sédiments et dynamique fluviale de la Magne. Mém. Lic. Sc. Géog., Univ. Liège, 168 p.

Poinsart D. (1992). - Effets des aménagements fluviaux sur les débits liquides et solides. L'exemple du Rhône dans les plaines de Miribel-Jonage et de Donzère-Mondragon. Thèse de Géographie et Aménagement, Univ. Lyon 3, 501 p.

Reid I. \& Frostick L.E. (1985). - Dynamics of bedload transport in Turkey Brook, a coarse-grained alluvial channel. Earth Surface Processes and Landforms, 11 : 143-155.

Van den Berght J.H. (1995). — Prediction of alluvial pattern of perennial streams. Geomorphology, 12, 259-279.

Williams G.P. (1983). - Paleohydrological methods and some examples from swedish fluvial environments. Geografiska Annaler, 65A (3-4), 227-243.

\section{NOTES}

1. Le critère adimensionnel de Shields (㯺) (cf. relation 3) permet de déduire la force tractrice critique $\left({ }_{59}{ }_{c}\right)$, c'est-à-dire la force tractrice nécessaire à la mise en mouvement d'un élément de

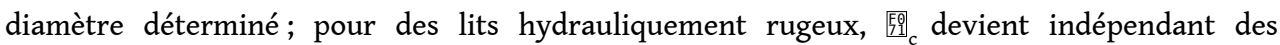
conditions de rugosité et tend à s'approcher d'une valeur constante égale à 0,060 . Toutefois, des valeurs plus faibles $(0,045$ voire 0,030$)$ ont souvent été avancées (cf. Bravard \& Petit, 1997) ; de plus, Andrews (1983) a montré que 㑌 ${ }_{\mathrm{c}}$ pouvait varier, notamment en fonction de la position des particules sur le lit (effet de saillie relative), ce qui s'est vérifié dans plusieurs autres études.

2. La relation de Meyer-Peter est très fréquemment utilisée pour estimer le débit solide transporté sous forme de charge de fond; elle se présente sous la forme suivante : $\mathrm{gb}=0,253$ (医9 - 医 $\left.{ }_{\mathrm{c}}\right)^{1,5}$ où $\mathrm{g}_{\mathrm{b}}$ est le transport de densité apparente sous eau par unité de temps et de largeur, 䜿 9 la force tractrice due à la résistance des grains (définie au $\S 2$ ) et ${ }_{54}{ }_{c}$ la force tractrice critique 
déduite du critère adimensionnel de Shields (cf. note 1). Cette méthode peut donner d'assez bons résultats à condition de bien déterminer les valeurs de [7ه $_{\mathrm{C}}$ (Bravard \& Petit, 1997).

3. Les step-pool systems représentent une forme de lit fluvial, à chenal unique et à méandration peu développée, où le profil longitudinal se caractérise par une succession généralement régulière de marches et de cuvettes se différenciant par l'importance des pentes du plan d'eau et la taille du matériel. Les marches résultent de l'alignement, perpendiculairement à l'écoulement, de blocs (mobilisables uniquement lors de crues exceptionnelles).

\section{RÉSUMÉS}

Des observations menées avec des cailloux marqués ont été effectuées dans rivières de dimension différente de l'Ardenne belge (taille du bassin variant de moins de $1 \mathrm{~km} 2$ à $2700 \mathrm{~km} 2$ ). La puissance spécifique a été évaluée pour les débits de mise en mouvement de la charge de fond, de sorte que des valeurs de puissances spécifiques critiques sont proposées. Trois types de relations liant la puissance spécifique critique $\left(\omega_{0}\right)$ à la taille des éléments mobilisés $\left(\mathrm{d}_{\mathrm{i}}\right)$ ont été mises en évidence. Pour la rivière la plus importante (l'Ourthe), les valeurs de $\omega_{0}$ sont les plus faibles et se rapprochent des valeurs proposées par Costa et par Williams. Dans les rivières de dimension intermédiaire (taille du bassin comprise entre 40 et $500 \mathrm{~km} 2$ ), les puissances spécifiques critiques sont plus élevées, ce qui résulte du fait qu'il existe une résistance des formes plus importante, cette dernière consommant une part accrue de l'énergie, au détriment de celle disponible pour la mobilisation et le transport de la charge de fond. L'importance de cette résistance des formes, qui peut être appréhendée par la force tractrice due à la résistance des formes ( $\tau$ "), ressort alors du rapport entre la force tractrice des particules $\left(\tau^{\prime}\right)$, la seule qui intervient dans la mobilisation et le transport de la charge de fond, et la force tractrice totale $(\tau)$; ce rapport varie entre 0,4 à 0,5 dans ces rivières intermédiaires contre 0,7 dans l'Ourthe. Dans les têtes de bassin (superficie inférieure à $20 \mathrm{~km} 2)$, où les pertes d'énergie dues à la résistance des formes sont encore plus élevées $\left(\tau^{\prime} / \tau<\right.$ $0,3)$, les puissances spécifiques critiques sont plus importantes que dans les deux autres types.

Experiments with marked pebbles were carried out on different sized rivers of the Belgian Ardenne (catchment areas varying from less than $1 \mathrm{~km} 2$ to $2,700 \mathrm{~km} 2$ ). Unit stream power required to cause bedload movement has been evaluated and critical values obtained. Three types of relationship between critical unit stream power $\left(\omega_{0}\right)$ and grain size $\left(d_{i}\right)$ have been established. The values for $\omega_{0}$ in the largest river (the Ourthe) were the lowest and were close to the values obtained by Costa and Williams. In rivers of intermediate size (catchment area between 40 and $500 \mathrm{~km} 2$ ), the critical stream power was higher due to greater resistance of the bedform. This resistance uses up some of the increase in energy that can cause movement and transport of bedload. The amount of resistance of the bedform can be expressed as bedform shear stress $\left(\tau^{\prime \prime}\right)$, determined by the relationship between grain shear stress $\left(\tau^{\prime}\right)$, that determines movement and transport of the bedload, and the total shear stress $(\tau)$. This ratio varies between 0.4 and 0.5 in the intermediate size rivers, compared to 0.7 in the Ourthe. In the higher catchment areas (less than $20 \mathrm{~km} 2)$, there is greater loss of energy due to bedform resistance $\left(\tau^{\prime} /\right.$ $\tau<0.3)$. In this third type, the specific critical funit stream power is higher than in the other two.

Beobachtungen mit markierten Kieseln wurden in Flüssen verschiedener Grösse in den belgischen Ardennen durchgeführt (Einzugsbereiche zwischen unter $1 \mathrm{~km} 2$ und $2700 \mathrm{~km} 2$ ). Die 
spezifische Kraft wurde für die bewegte Menge der Grundfracht ermittelt, so dass spezifische kritische Kräftewerte vorgeschlagen werden können. Drei Typen der Beziehung zwischen spezifischer kritischer Kraft $\left(\omega_{0}\right)$ und der Menge der bewegten Elemente $\left(d_{i}\right)$ werden deutlich gemacht. Bei dem wichtigsten Fluss, der Ourthe, sind die Werte für $\omega_{0}$ am geringsten und nähern sich den von Costa und Williams ermittelten Werten. Bei den Flüssen der mittleren Dimension (Einzugsbereich zwischen 40 und $500 \mathrm{~km} 2$ ) sind die spezifischen kritischen Kräfte grösser, da der Widerstand der Formen höher ist, mit einem gesteigerten Energieverbrauch auf Kosten der für die Mobilisierung und den Transport der Grundfracht verfügbaren Energie. Die Bedeutung dieses Formenwiderstands, der als « bedform shear stress » ( $\tau$ ") erfasst werden kann, ergibt sich aus der Beziehung zwischen dem "grain shear stress » $\left(\tau^{\prime}\right)$, der sich in Mobilisierung und Transport der Grundfracht auswirkt, und dem «total shear stress » $(\tau)$. Diese Beziehung variiert zwischen 0,4 und 0,5 bei den mittelgrossen Flüssen gegenüber 0,7 bei der Ourthe. Bei den Einzugsbereichen unter $20 \mathrm{~km} 2$, wo die Energieverluste infolge des Formenwiderstandes noch höher liegen $\left(\tau^{\prime} / \tau<\right.$ $0,3)$, sind die spezifischen kritischen Kräfte stärker als bei den beiden anderen Typen.

\section{INDEX}

Keywords : Ardenne, critical unit stream power, flow, flow rate, gravel-bed rivers

Mots-clés : Ardenne, débit, écoulement, puissance spécifique critique, rivières à charge de fond caillouteuse

Schlüsselwörter : Abfluss, Abflussmenge, Ardennen, Grundfracht, spezifische kritische Kraft

\section{AUTEURS}

\section{FRANÇOIS PETIT}

Département de Géographie physique - Université de Liège - Allée du 6 août, 2 (Bât. B11), Sart Tilman B4000 - Liège (Belgique)

\section{GEOFFREY PERPINIEN}

Département de Géographie physique - Université de Liège - Allée du 6 août, 2 (Bât. B11), Sart Tilman B4000 - Liège (Belgique)

\section{CARL DEROANNE}

Département de Géographie physique - Université de Liège - Allée du 6 août, 2 (Bât. B11), Sart Tilman B4000 - Liège (Belgique) 